\title{
Teknokultura
}

ISSNe: 1549-2230

http://dx.doi.org/10.5209/TEKN.59821

\section{Andrea. Red de confianza, acompañamiento y acción contra la violencia machista}

Jordi Claramonte'; Lucia Gutiérrez $^{2}$

Recibido: 9 de abril de 2018 / Revisado: 29 de junio de 2018 / Aceptado: 18 de septiembre de 2018 Open peer reviews

Resumen. Andrea es un proyecto para construir redes de confianza, acompañamiento y acción para mujeres amenazadas por la violencia machista. Una plataforma que se plantea como una manera de facilitar que todas y cada una de las personas que queremos implicarnos contra las violencias machistas podamos hacer algo significativo. Auto-organizándonos, formándonos cuando sea preciso $\mathrm{y}$ atendiendo los problemas de manera directa, sin tener que depender de las determinaciones de las vías jurídicas, policiales y administrativas cuyas insuficiencias y contra-productividades se han hecho dolorosamente evidentes. Una red de apoyo permanente, que se posiciona ante un problema que nos toca a todas y todos.

Palabras clave: auto-organización; comunidad; feminicidio; maltrato; patriarcado.

\section{[en] Andrea Network of trust, accompaniment and action against sexist violence}

Abstract. Andrea is a project devoted to producing networks of trust, accompaniment and action for women threatened by gender-based violence. A platform which should facilitate that every person willing to do "something" can actually do it in a most significant way. Self-organizing, learning and working directly with the problems and their victims, without having to depend upon the limited resources and agendas of judges, police forces and social services which limitations and counter-productivities have become painfully obvious. Andrea stands for a permanent support network addressing a huge problem that does affect us all.

Keywords: abuse; community; feminicide; patriarchy; self-organizing.

Sumario. 1. Andrea. Red de confianza, acompañamiento y acción contra la violencia machista. 2. La historia de La Mina. 3. Andrea. 4. Necesidad y contexto. 5. Arquitectura de la red y tareas en marcha. 6. Infraestructura de la red. 7. Algunas consideraciones adicionales. 8. Coda. 9. Agradecimientos. 10. Referencias.

Cómo citar: Claramonte, J.; Gutiérrez, L. (2018). Andrea. Red de confianza, acompañamiento y acción contra la violencia machista, en Teknokultura 15(2), 339-355.

\footnotetext{
$1 \quad$ UNED (España)

jclaramonte@fsof.uned.es

2 Arquitecta (España)

gutierrez.vazquez.lucia@gmail.com
} 


\section{Andrea. Red de confianza, acompañamiento y acción contra la violencia machista}

Andrea Carballo Claramonte tenía 20 años recién cumplidos. El día 23 de diciembre de 2017 fue asesinada por su expareja, que estrelló contra una gasolinera el coche en el que la había forzado a subir. Andrea le había denunciado por malos tratos días antes. Él tenía antecedentes y una orden de alejamiento de su anterior pareja. Ya había intentado atropellar a Andrea y había pinchado las ruedas del coche de la madre de Andrea. El caso fue catalogado como "riesgo medio" y se determinó una orden de alejamiento de 200 metros.

Es inevitable plantearse qué hubiera pasado si Andrea hubiera salido a trabajar acompañada de un grupo de personas la mañana del 23 de diciembre. ¿Y si los vecinos que sabían que "El Peonza" andaba rondando la zona hubieran tenido medios eficaces y rápidos para avisarla, para organizarse, para evitar que la metiera en el coche? ¿Y si para denunciar que una orden de alejamiento de 200 metros no sirve, Andrea y su familia hubieran podido contar con el apoyo de una red más numerosa y más capaz de movilizarse teniendo acceso a medios de comunicación y otras redes sociales y vecinales?

Figura 1. Andrea Carballo Claramonte. Fuente: Elespañol.com

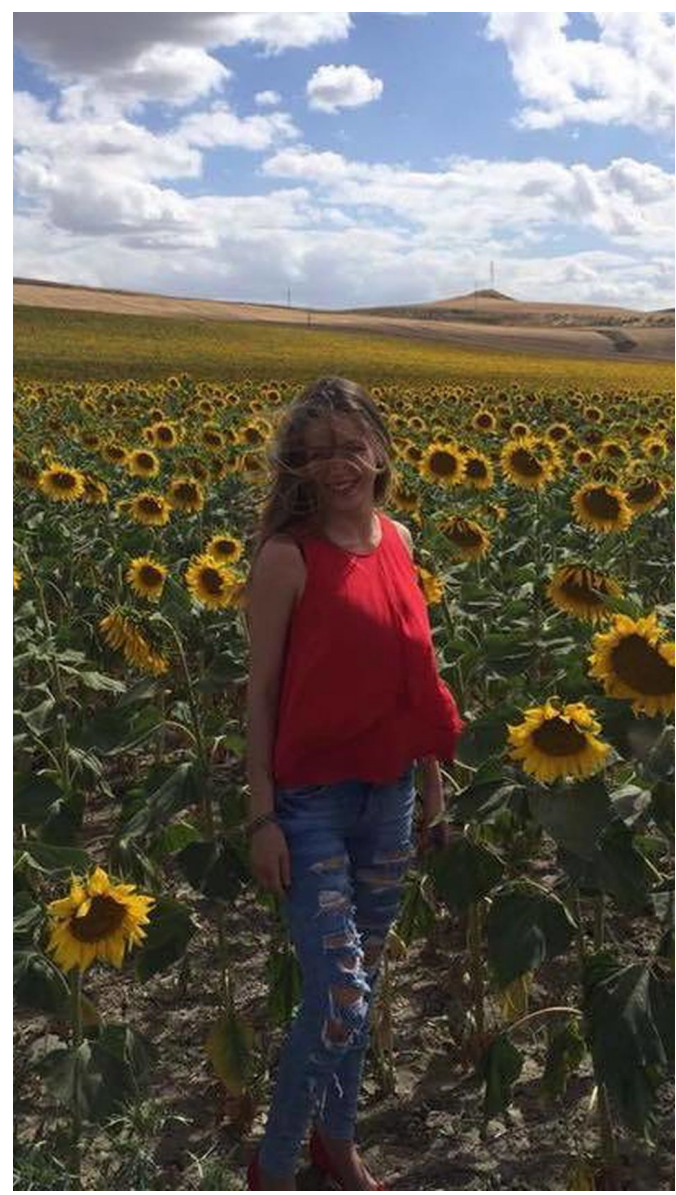


La violencia contra las mujeres está muy lejos de ser un problema personal entre agresor y víctima. Por eso nos tocan a todas, nos tocan a todos. La historia de Andrea, como la de todas las mujeres acosadas, amenazadas y agredidas, es por entero un problema político. Como señala Ana de Miguel Álvarez en su artículo La construcción de un marco feminista de interpretación: la violencia de género (2005), se trata de una situación de violencia estructural -del orden patriarcal-sobre el colectivo femenino.

Según la ONU, el maltrato a la mujer es el crimen más numeroso del mundo. Recoge Diana Fernández Romero en su Tesis Doctoral Destrucción y Reconstrucción de la Identidad de la mujeres maltratadas: Análisis de discursos autobiográficos y de publicidad institucional (2012) que al menos una de cada tres mujeres en todo el mundo ha sido golpeada, coaccionada sexualmente o abusada durante su vida, según el Fondo de Desarrollo de las Naciones Unidas para la Mujer $(\mathrm{UNWOMEN})^{3}$. El Consejo de Europa declaró en 2002 que la violencia contra la mujer es la causa mayor de muerte y discapacidad de las mujeres de entre 16 y 44 años, por encima de las guerras, los accidentes de tráfico y el cáncer. La violencia machista contra las mujeres tiene, sin duda, muchas caras, como se desprende del informe de UNIFEM (hoy UNWOMAN) Ni un Minuto Más: Poner Fin a la Violencia contra la Mujer, según el cual una de cada cinco mujeres se convertirá en víctima de violación o intento de violación. Que entre 100 y 140 millones de mujeres y niñas a nivel mundial han sido sometidas a ablación o mutilación genital, y unas 800.000 personas, el $80 \%$ de ellas mujeres, son objeto de tráfico sexual. En este proyecto, sin embargo, y por dolorosas e insoportables que puedan ser todas esas formas de violencia, nos tendremos que centrar en la violencia que se genera en el ámbito de la relación de pareja, ya se trate de una pareja en la que haya convivencia o una en la que la relación se haya suspendido.

En España, de las mujeres por encima de los quince años, el 10,4\% ha sufrido violencia física, el $8,1 \%$ violencia sexual y el $25,4 \%$ violencia psicológica por parte de alguna pareja o expareja en algún momento de su vida, según la Macroencuesta de Violencia contra la Mujer de 2015, elaborada por el Ministerio de Sanidad, Servicios Sociales e Igualdad. Desde el año 2003 - primer año del que encontramos estadísticas registradas - han sido 939 las víctimas mortales por violencia machista, según las cifras oficiales del Gobierno (Ministerio de la Presidencia, s/f). Sólo en 2017 los juzgados españoles recibieron un total de 166.620 denuncias por violencia de género, según las cifras del Observatorio contra la Violencia Doméstica y de Género del Consejo General del Poder Judicial (CGPJ). Las sentencias dictadas fueron 49.165, de las que 33.146 fueron condenas (menos del 20\% del total de denuncias) y 16.019, absoluciones.

\section{La historia de La Mina}

Pocos días después de saber del asesinato de Andrea, leíamos en los periódicos una historia que sería clave, comentando el feminicidio de Kenya Álvarez sucedido el día de Nochebuena en la barrio barcelonés de La Mina. Un miembro de la comunidad gitana argumentaba que las mujeres gitanas no eran asesinadas por sus parejas o

3 http://unwomen.org 
exparejas, porque, al contrario que las payas, ellas contaban una familia muy grande que las acogía y respaldaba.

Con independencia del grado de veracidad que dicha afirmación pudiera tener en el caso concreto de las mujeres de etnia gitana, había en el pronunciamiento varios ingredientes de indudable lucidez:

- $\quad$ En un primer momento, la lucidez de reconocer la gravedad de una de las fases fundamentales de todo maltrato, la que consiste en el aislamiento de la mujer, en la ruptura de los lazos que ésta pudiera mantener con familiares y amigos/as.

- $\quad$ En un segundo momento, se trataba de advertir que para romper el bucle de malos tratos y violencia las mujeres iban a necesitar algo parecido a una familia capaz de ofrecer respaldo incondicional.

La cuestión ahí se volvía obvia ¿cómo haremos para generar esa "familia"?

$\mathrm{O}$ dicho de otro modo, y por no recurrir a un término tan lastrado como el de familia, ¿por qué no nos organizamos para tramar redes de apoyo permanente a mujeres que se vean amenazadas? Grupos de personas con diferentes perfiles que estén disponibles y conectadas con las mujeres amenazadas, en tiempo real.

Eso es Andrea.

\section{Andrea}

Figura 2. Plataforma Andrea. Fuente: elaboración propia.

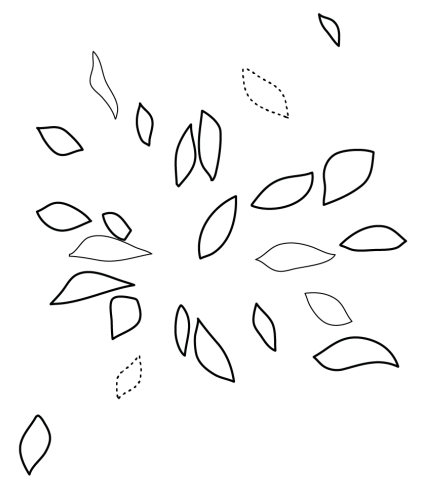

personas que queremos "hacer algo" inscritas en la red

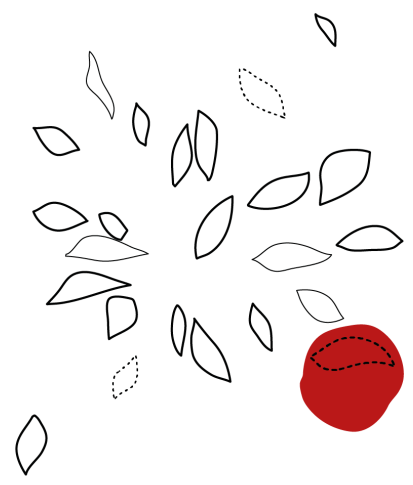

mujer amenazada

Andrea es un proyecto para construir redes de apoyo para mujeres amenazadas por la violencia machista.

Porque la violencia machista no es un problema que tengan algunas mujeres. Es un problema que tenemos todos y todas. Y frente al que todos o todas tenemos que posicionarnos. Y no hacer nada - obviamente- es ya tomar una posición... 
Para ello empezaremos ofreciendo la posibilidad de que cualquier persona que quiera implicarse pueda hacerlo a través de un gesto tan sencillo como darse de alta en la página web que da sustento a la plataforma. Bastará con que proporciones tu nombre, tu código postal y tu número de teléfono móvil.

A través de esta web tendrás acceso a foros, cursos de formación y sobre todo formarás parte de una comunidad dispuesta a movilizarse en cuanto se registre una amenaza en una ubicación cercana a tu domicilio habitual. Podrás incluir en tu perfil, si quieres, información sobre ti, tu disponibilidad, o aquello que puedes aportar a la red de manera más concreta.

En cuanto una mujer amenazada solicite apoyo, la plataforma le asignará un grupo de personas que vivan y se muevan cerca de ella para que puedan formar su propia red de apoyo. Esta red de apoyo propia y personalizada de cada mujer funcionará como un satélite de la web, con un servicio BOT, similar a un grupo de Telegram.

Es de vital importancia que estas redes funcionen en tres planos diferentes y articulados entre sí: confianza, acompañamiento y acción.

\subsection{Confianza}

Figura 3. Confianza. Fuente: elaboración propia.

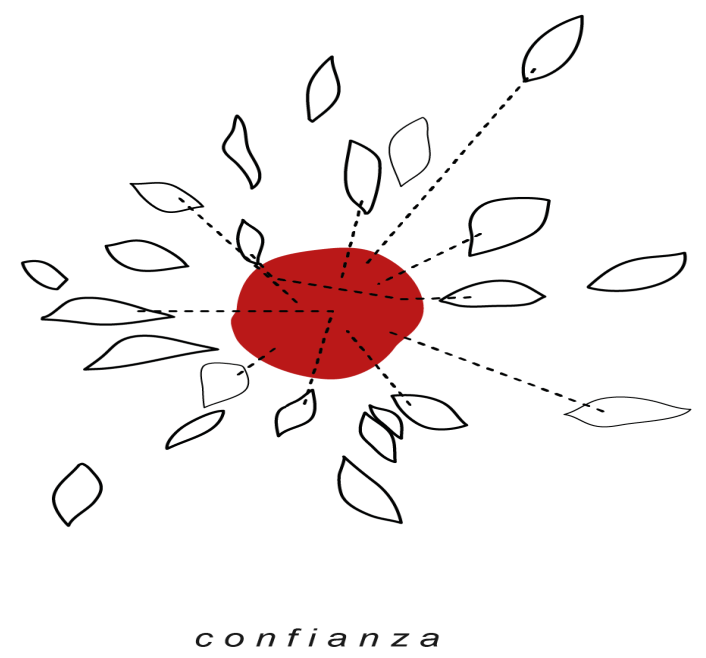

En primer lugar se tratará de establecer un grupo de referencia, cuyo control debe estar siempre en manos de la mujer amenazada. Ella deberá confirmar que confía en cada una de las personas que le haya asignado la plataforma, pudiendo recibir nuevas propuestas si por cualquier razón decide rechazar el apoyo de alguien. Este punto es absolutamente crucial para asegurarnos, por ejemplo, de que el maltratador no se infiltra en la red de confianza. Para ello habilitaremos opciones de bloqueo de números de teléfono y direcciones IP. Sabemos que ninguna medida es perfectamente segura en este aspecto, pero pensamos que estando en la red la mujer amenazada podrá hacer frente a estas situaciones de acoso y violencia desde unas condiciones mucho mejores. 
Se trata en esta fase de que la mujer amenazada empiece a recobrar la confianza en sí misma y en todas y cada una de las personas que forman su red. El proceso de recuperación de confianza es un proceso largo sin duda, pero es un proceso que es imprescindible iniciar ofreciendo justo aquello que el maltratador se ha encargado de eliminar: redes de afectos, referentes de confianza y un entorno desintoxicado.

Prácticamente la mitad de las mujeres asesinadas ni tan siquiera llegan a presentar denuncia alguna. Esto en gran medida puede deberse a la falta de confianza en la capacidad real de las instituciones para ofrecer ayuda solvente, y lo que es más grave, a la falta de confianza de las mujeres en su propia capacidad para desafiar la amenaza a la que se enfrentan.

Multitud de estudios muestran que el restablecimiento de esta confianza en modo alguno puede dejarse en manos de la policía o los servicios sociales:

A pesar de los servicios institucionales disponibles, los datos de la encuesta anual de percepción de la opinión sobre violencia de género, realizada en 2011 a instancias del Ministerio de Sanidad, Política Social e Igualdad, ponen de relieve que el $50 \%$ de las mujeres interrogadas cree que acudiría a un/a familiar en caso de sufrir malos tratos por parte de su pareja o expareja. El 29\% iría a la Policía o a un Juzgado, y el 9,5\% recurriría a los servicios sociales y sanitarios (Fernández Romero, 2012, p. 608).

Semejante escepticismo hacia la sensibilidad de jueces y policías quizás tenga mucho que ver con la percepción y experiencia generalizada de las mujeres que han intentado buscar ayuda. En su tesis, Fernández Romero recoge entre 2002 y 2008 testimonios de mujeres (27-60 años) que eran o habían sido maltratadas por sus parejas o exparejas y que estaban recibiendo ayuda en un centro institucional. En estas entrevistas, muchas de ellas destacaban que fueron las instituciones y los jueces los que las enjuiciaron, poniendo en cuestión la veracidad de sus testimonios, terminando en ocasiones por culpabilizarlas a ellas frente a la responsabilidad del maltratador.

Es por ello que esta confianza de la que hablamos necesita funcionar en doble sentido: que las mujeres amenazadas sepan que pueden confiar en las personas que tienen cerca, y que a su vez sientan, y así sea, que ese entorno confía en ellas, en la verdad de lo que han vivido y cuentan.

Muestran las entrevistas que las principales causas por las que muchas mujeres no compartían su situación con nadie eran la vergüenza y el miedo por la incomprensión, la incredulidad o el estigma social que pensaban podría conllevar el hecho de dar a conocer su situación y denunciarla. Sin embargo, las que lo hicieron reconocen que el respaldo que se les ofreció fue esencial para poder ser y hacer, para poder entender lo que estaba sucediendo, recuperar la autoestima y adquirir nuevas herramientas.

Esta fase de la red tiene que servirle a la mujer amenazada para cerciorarse de que no está sola y de que si decide denunciar va a contar con todo el apoyo que necesite. 


\subsection{Acompañamiento}

Figura 4. Acompañamiento. Fuente: elaboración propia.
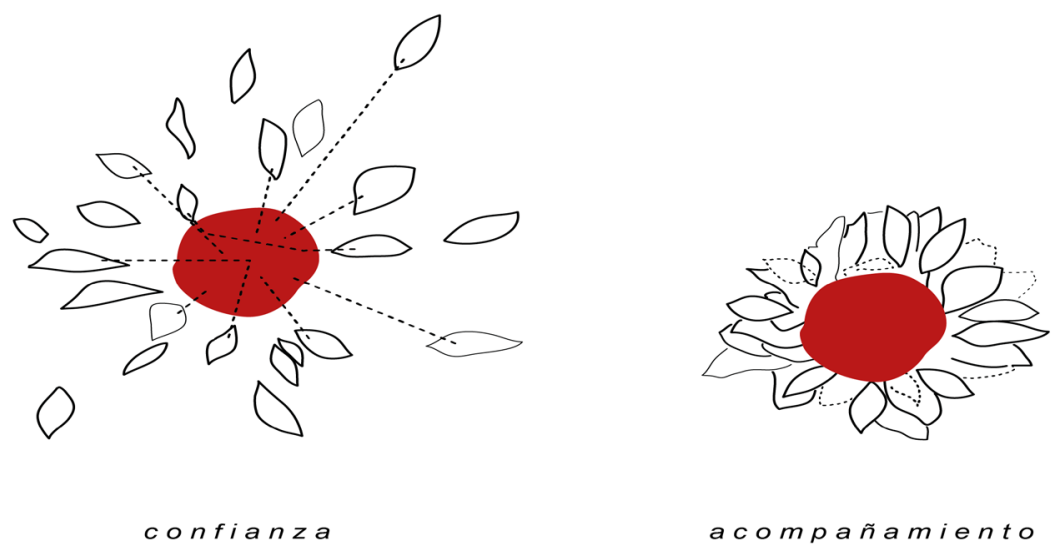

acompañamiento

En el momento en que cada mujer así lo decida, la red empezará con tareas de acompañamiento. Puede tratarse de algo aparentemente tan trivial -y tan fundamental- como no estar sola en momentos en que pueda sentirse más frágil: al salir de casa para ir al trabajo o al volver del mismo, al llevar a los/as niños/as a la escuela, al ir a realizar cualesquiera gestiones o denuncias. Esta será una de las funciones clave de la red de apoyo y deberá implicar a tantas personas como sea menester, puesto que el acoso es uno de los patrones más insidiosos y habituales:

Una vez se separaban, el agresor las sometía a un persistente acoso y a una constante persecución por no cumplir el "contrato" de amor eterno y entregado y por falsear su condición de mujer que lo da todo por amor. Era ese hostigamiento el que, según las mujeres, les impedía deshacerse completamente del maltratador y ralentizaba la conquista de sus nuevos objetivos, de tal forma que en ocasiones llegaban a arrepentirse de haber dado el paso de romper (Fernández Romero, 2012, p.387).

El acompañamiento puede darse en el entorno habitual de la mujer amenazada o puede también consistir en ayudarla a encontrar y afianzar otro entorno en el caso en que la mujer desee cambiar de barrio o incluso de ciudad. Y es que otra de las razones principales por las que muchas mujeres no se atreven a denunciar es el pensar que hacerlo supondría un problema de seguridad para ellas y sus hijos/as, y que después tendrían que regresar a su domicilio a convivir con el maltratador al menos un tiempo.

Acompañamiento puede ser también el saberse conectada de manera permanente a través de Internet con personas de confianza. Saber que en todo momento hay gente al otro lado que responde, está pendiente y dispuesta a salir en cualquier circunstancia.

Vaya donde vaya no tendrá que estar sola si no lo desea. 


\subsection{Acción}

Figura 5. Acción. Fuente: elaboración propia.
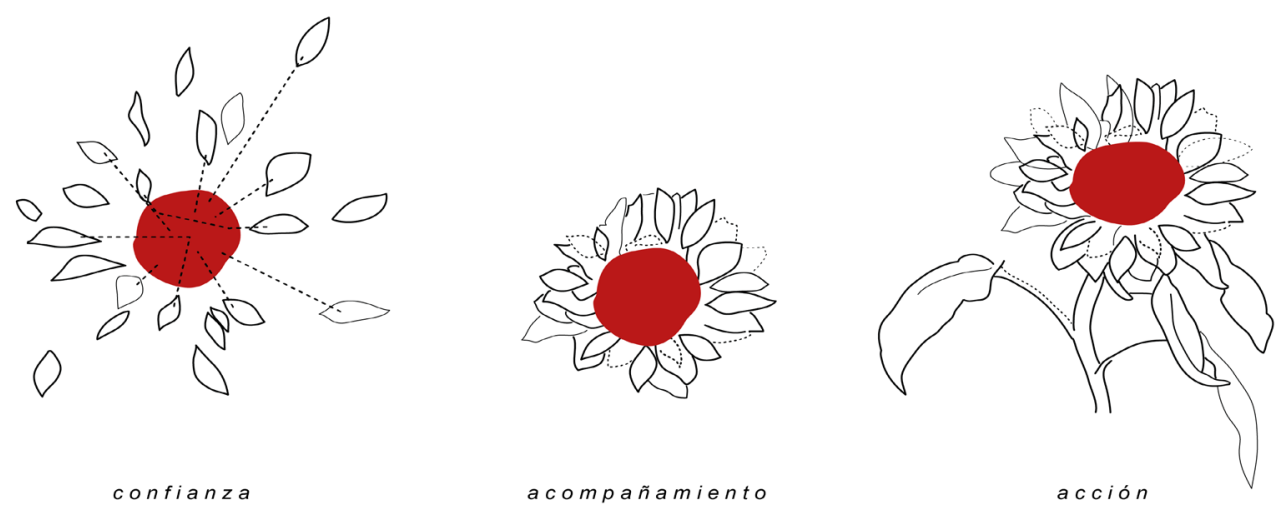

Más allá de la confianza y el acompañamiento, entendemos que la red puede implicarse, si la mujer amenazada así lo estima conveniente, movilizándose públicamente, exigiendo mejoras en las medidas de protección judicial y policial. En este sentido, habrá que organizar pequeñas campañas tácticas que puedan servir para dar visibilidad a cada caso y sobre todo a la insuficiencia de la cobertura institucional.

Otra veta de acción importante puede consistir - siempre que la mujer así lo aprecie- en dar a conocer el caso a más vecinas y vecinos que puedan a su vez implicarse en una especie de red informal que ayude a asegurar, por ejemplo, que se cumplen las órdenes de alejamiento. Si las personas que llevan la panadería, el quiosco de prensa o la portería de los edificios vecinos conocen la situación y saben a quién deben llamar si ven algo raro, podemos tener muchas más posibilidades de mantener a salvo la vida de la mujer amenazada... pero sobre todo conseguiremos que la violencia machista deje de ser un problema personal y cobre la dimensión social que en verdad tiene.

Recuperar la autonomía, la voz, la capacidad de obrar y comprender... para ser, hacer y estar es una dirección importantísima aquí para la mujer amenazada. La fase de acción puede servir para que ella, en vez de aceptarse apocada, se convierta en una dinamizadora de su red y en agente fundamental de otras redes de apoyo que puedan surgir y de la que ella pueda ahora ser parte también. 


\section{Necesidad y contexto}

Andrea se plantea como una manera de facilitar que todas y cada una de las personas que queremos implicarnos contra las violencias machistas podamos hacer algo significativo. Auto-organizándonos, formándonos cuando sea preciso $\mathrm{y}$ atendiendo los problemas de manera directa, sin tener que depender de las determinaciones de las vías jurídicas, policiales y administrativas cuyas insuficiencias y contra-productividades han sido puestas de manifiesto repetidamente: "También fueron críticas con el sistema de ayudas, pues sostenían que éstas no siempre llegaban, eran insuficientes y no protegían debidamente a las mujeres" (Fernández Romero, 2012, p. 378).

De las víctimas mortales por violencia de género del pasado año 2017 en España, el $22,4 \%$ de las mujeres -casi la cuarta parte- habían denunciado al agresor. El $12,2 \%$ fueron asesinadas teniendo medidas de protección en vigor, según el Ministerio de Sanidad, Servicios Sociales e Igualdad. De acuerdo a la Macroencuesta de 2015 , el $86,7 \%$ de las mujeres que están sufriendo violencia de género han acudido a la policía y al juzgado.

En 2009 el Estado invirtió en 3.000 pulseras de control telemático para localizar permanentemente por GPS a los agresores con orden de alejamiento. Este año se revelaba que el $73 \%$ no se han usado.

Para las mujeres que cuentan con una Orden de Protección o medida de alejamiento vigente, se ofrece desde el Estado un programa de teleasistencia móvil (ATENPRO). Sin embargo, dejan fuera a gran parte de las mujeres, pues como requisitos para obtener esta ayuda se exige que no se conviva con el maltratador y que se participe en los programas de atención especializada de la comunidad autónoma correspondiente.

Se quedan fuera también las mujeres amenazadas que por cualquier motivo no quieran o puedan acercarse a la policía o a las instituciones judiciales. Es el caso de las mujeres migrantes en situación "irregular", que según el informe de 2011 de Amnistía Internacional (Fernández Romero, 2012, p. 88) no denuncian por temor a ser expulsadas del país de acogida si se descubre su situación ilegal.

Se hace evidente que ni los plazos marcados por la vía judicial ni los recursos proporcionados por la administración y la policía son suficientes. Tampoco lo son sus criterios.

Fui con mi abogada, no me cogieron la denuncia (...) Otra vez fui con mi amiga y no me cogieron la denuncia. El maltrato psicológico no es maltrato (Gloria) (Fernández Romero, 2012, p. 378).

¿Cómo es posible que sin parte de lesiones no se acepte la denuncia por malos tratos?

Estamos planteando la plataforma Andrea ante la evidente necesidad de otras vías de pensar, para poner en marcha otras formas de actuación, que no se vean supeditadas a los tiempos, medidas y lenguaje de las instituciones. 
Tabla 1. Lenguaje y categorización. Fuente: Ministerio del Interior.

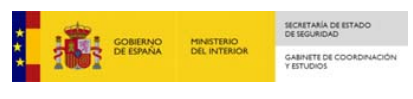

CASOS DE VIOLENCIA DE GÉNERO

DISTRIBUCIÓN TERRITORIAL

País nacimiento víctima: Todos los países

(Datos a 31/10/2017)

Edad víctima: Todas las edades

\begin{tabular}{|c|c|c|c|c|c|c|c|c|c|c|c|}
\hline \multicolumn{12}{|c|}{ Sistema de Seguimiento Integral en los casos de Violencia de Género (Sistema VioGén) } \\
\hline \multirow{2}{*}{$\begin{array}{l}\text { COMUNIDAD AUTÓNOMA / } \\
\text { PROVINCIA }\end{array}$} & \multicolumn{5}{|c|}{$\begin{array}{l}\text { NIVEL DE RIESGO } \\
\text { (Casos activos) }\end{array}$} & \multicolumn{3}{|c|}{ CASOS } & \multirow{2}{*}{ VÍCTIMAS } & \multirow{2}{*}{$\begin{array}{c}\text { PSP } \\
\text { desde } \\
17 / 08 / 2016\end{array}$} & \multirow{2}{*}{$\begin{array}{l}\text { Gestión } 3 A \\
\text { desde } \\
15 / 02 / 2017\end{array}$} \\
\hline & $\begin{array}{c}\text { No } \\
\text { apreciado }\end{array}$ & Bajo & Medio & Alto & Extremo & ACtIVos & INACTIVOS & TOTAL & & & \\
\hline Almería & 1.019 & 576 & 67 & 2 & & 1.664 & 8.570 & 10.234 & 9.546 & 1.663 & 1.521 \\
\hline Cádiz & 996 & 1.332 & 195 & 8 & & 2.531 & 12.703 & 15.234 & 14.075 & 2.642 & 4.213 \\
\hline Córdoba & 299 & 461 & 145 & 6 & & 911 & 5.802 & 6.713 & 6.255 & 1.398 & 1.061 \\
\hline Granada & 659 & 562 & 240 & 8 & & 1.469 & 9.804 & 11.273 & 10.526 & 2.141 & 1.426 \\
\hline Huelva & 532 & 448 & 89 & 5 & 1 & 1.075 & 4.818 & 5.893 & 5.536 & 1.486 & 897 \\
\hline Jaén & 578 & 611 & 133 & 5 & 1 & 1.328 & 4.975 & 6.303 & 5.918 & 1.725 & 1.165 \\
\hline Málaga & 1.659 & 1.204 & 225 & 7 & & 3.095 & 18.008 & 21.103 & 19.581 & 3.505 & 2.399 \\
\hline Sevilla & 1.515 & 1.413 & 275 & 7 & 3 & 3.213 & 19.047 & 22.260 & 20.786 & 4.125 & 2.069 \\
\hline Andalucía & 7.257 & 6.607 & 1.369 & 48 & 5 & 15.286 & 83.727 & 99.013 & 92.223 & 18.685 & 14.751 \\
\hline Huesca & 162 & 75 & 15 & 1 & & 253 & 1.429 & 1.682 & 1.569 & 176 & 126 \\
\hline Teruel & 49 & 46 & 17 & 1 & & 113 & 750 & 863 & 806 & 172 & 101 \\
\hline Zaragoza & 367 & 662 & 96 & 6 & & 1.131 & 9.164 & 10.295 & 9.559 & 1.949 & 1.563 \\
\hline Aragón & 578 & 783 & 128 & 8 & & 1.497 & 11.343 & 12.840 & 11.934 & 2.297 & 1.790 \\
\hline Asturias & 644 & 494 & 97 & 3 & & 1.238 & 9.208 & 10.446 & 9.558 & 1.840 & 1.526 \\
\hline Islas Baleares & 1.295 & 803 & 224 & 5 & & 2.327 & 15.215 & 17.542 & 16.195 & 2.291 & 2.627 \\
\hline \begin{tabular}{|l|} 
Las Palmas \\
\end{tabular} & 687 & 981 & 98 & 5 & 1 & 1.772 & 14.162 & 15.934 & 14.774 & 2.330 & 3.099 \\
\hline Santa Cruz de Tenerife & 838 & 952 & 87 & 1 & & 1.878 & 13.685 & 15.563 & 14.430 & 2.011 & 1.276 \\
\hline $\begin{array}{r}\text { Canarias } \\
\end{array}$ & 1.525 & 1.933 & 185 & 6 & 1 & 3.650 & 27.847 & 31.497 & 29.204 & 4.341 & 4.375 \\
\hline Cantabria & 326 & 295 & 70 & 6 & & 697 & 4.502 & 5.199 & 4.900 & 790 & 1.594 \\
\hline Ávila & 84 & 94 & 15 & & & 193 & 1.182 & 1.375 & 1.269 & 242 & 86 \\
\hline Burgos & 220 & 189 & 33 & 3 & & 445 & 2.599 & 3.044 & 2.826 & 590 & 386 \\
\hline León & 271 & 227 & 62 & 1 & & 561 & 3.191 & 3.752 & 3.493 & 834 & 716 \\
\hline Palencia & 102 & 78 & 12 & & & 192 & 1.075 & 1.267 & 1.181 & 218 & 207 \\
\hline Salamanca & 234 & 86 & 9 & 1 & & 330 & 2.367 & 2.697 & 2.541 & 553 & 285 \\
\hline Segovia & 45 & 115 & 26 & 1 & & 187 & 1.097 & 1.284 & 1.231 & 202 & 164 \\
\hline Soria & 76 & 33 & 3 & 1 & & 113 & 617 & 730 & 700 & 145 & 78 \\
\hline Valladolid & 379 & 83 & 15 & 1 & 1 & 479 & 3.643 & 4.122 & 3.860 & 786 & 1.387 \\
\hline Zamora & 91 & 39 & 7 & 2 & & 139 & 1.208 & 1.347 & 1.258 & 216 & 249 \\
\hline Castilla y León & 1.502 & 944 & 182 & 10 & 1 & 2.639 & 16.979 & 19.618 & 18.359 & 3.786 & 3.558 \\
\hline Albacete & 357 & 191 & 21 & 3 & & 572 & 3.223 & 3.795 & 3.520 & 469 & 1.206 \\
\hline Ciudad Real & 351 & 251 & 63 & 2 & 2 & 669 & 4.651 & 5.320 & 4.948 & 922 & 998 \\
\hline Cuenca & 201 & 42 & 7 & 1 & & 251 & 1.486 & 1.737 & 1.618 & 275 & 375 \\
\hline Guadalajara & 183 & 135 & 47 & 1 & 1 & 367 & 2.412 & 2.779 & 2.612 & 427 & 264 \\
\hline Toledo & 663 & 274 & 46 & 3 & & 986 & 5.529 & 6.515 & 6.114 & 989 & 563 \\
\hline \begin{tabular}{|c|} 
Castilla-La Mancha \\
\end{tabular} & 1.755 & 893 & 184 & 10 & 3 & 2.845 & 17.301 & 20.146 & 18.812 & 3.082 & 3.406 \\
\hline Barcelona & & & & & & & 2.832 & 2.832 & 2.674 & 121 & 479 \\
\hline Girona & & & & & & & 324 & 324 & 294 & 18 & 103 \\
\hline Lleida & & & & & & & 273 & 273 & 246 & 11 & 140 \\
\hline Tarragona & & & & & & & 2.218 & 2.218 & 2.164 & 25 & 59 \\
\hline Cataluña (1) & & & & & & & 5.647 & 5.647 & 5.378 & 175 & 781 \\
\hline Alicante & 2.000 & 1.505 & 348 & 18 & & 3.871 & 21.701 & 25.572 & 23.553 & 4.198 & 2.421 \\
\hline Castellón & 350 & 377 & 70 & 3 & & 800 & 5.641 & 6.441 & 5.960 & 1.025 & 722 \\
\hline Valencia & 2.119 & 1.595 & 253 & 8 & & 3.975 & 27.840 & 31.815 & 29.079 & 5.164 & 2.940 \\
\hline $\begin{array}{c}\text { Comunidad Valenciana } \\
\end{array}$ & 4.469 & 3.477 & 671 & 29 & & 8.646 & 55.182 & 63.828 & 58.592 & 10.387 & 6.083 \\
\hline Badajoz & 390 & 371 & 158 & 5 & & 924 & 4.893 & 5.817 & 5.424 & 811 & 1.108 \\
\hline Cáceres & 341 & 257 & 53 & 1 & & 652 & 2.588 & 3.240 & 3.034 & 414 & 1.130 \\
\hline Extremadura & 731 & 628 & 211 & 6 & & 1.576 & 7.481 & 9.057 & 8.458 & 1.225 & 2.238 \\
\hline A Coruña & 504 & 714 & 135 & 6 & & 1.359 & 7.902 & 9.261 & 8.624 & 1.316 & 2.546 \\
\hline Lugo & 258 & 170 & 31 & & & 459 & 2.455 & 2.914 & 2.741 & 696 & 516 \\
\hline Ourense & 236 & 121 & 15 & & & 372 & 2.311 & 2.683 & 2.504 & 483 & 407 \\
\hline Pontevedra & 518 & 370 & 67 & 2 & & 957 & 8.910 & 9.867 & 9.147 & 1.389 & 1.420 \\
\hline Galicia & 1.516 & 1.375 & 248 & 8 & & 3.147 & 21.578 & 24.725 & 23.016 & 3.884 & 4.889 \\
\hline Madrid & 4.379 & 2.698 & 608 & 24 & & 7.709 & 64.139 & 71.848 & 67.343 & 11.136 & 6.093 \\
\hline Murcia & 1.129 & 1.175 & 234 & 4 & & 2.542 & 15.820 & 18.362 & 17.088 & 2.730 & 1.820 \\
\hline Navarra & 471 & 323 & 58 & 2 & & 854 & 3.797 & 4.651 & 4.376 & 666 & 488 \\
\hline Álava & & & & & & & 200 & 200 & 186 & 9 & 27 \\
\hline Guipúzcoa & & & & & & & 247 & 247 & 234 & 6 & 141 \\
\hline Vizcaya & & & & & & & 593 & 593 & 542 & 28 & 71 \\
\hline País Vasco (1) & & & & & & & 1.040 & 1.040 & 962 & 43 & 239 \\
\hline La Rioja & 161 & 140 & 73 & 6 & & 380 & 2.671 & 3.051 & 2.858 & 599 & 370 \\
\hline Ceuta & 78 & 67 & 11 & & & 156 & 1.041 & 1.197 & 1.142 & 179 & 166 \\
\hline Melilla & 53 & 64 & 13 & & & 130 & 1.404 & 1.534 & 1.458 & 151 & 234 \\
\hline TOTAL & 27.869 & 22.699 & 4.566 & 175 & 10 & 55.319 & 365.922 & 421.241 & 391.856 & 68.287 & 57.028 \\
\hline \multirow{3}{*}{\multicolumn{4}{|c|}{ Casos Mossos d'Esquadra enviados (vía servicios web) desde Cataluña }} & & & \multicolumn{2}{|c|}{ CASOS BAJA } & 52.593 & & & \\
\hline & & & & & & CASOS E & TRANJERO & 4.837 & & & \\
\hline & & & & 49.601 & & TOTAL & CASOS & 478.671 & & & \\
\hline
\end{tabular}

(1) Los casos inactivos de Cataluña y el País Vasco reflejan el número de víctimas que, estando recogidas en el Sistema VioGén (en otra parte del territorio nacional), en

la actualidad residen en alguna de estas dos Comunidades Autónomas. 


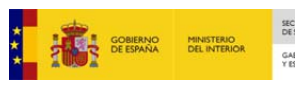

\begin{tabular}{|c|c|c|c|c|c|c|c|c|c|c|c|}
\hline INSTITUCIONES & $\begin{array}{c}\text { No } \\
\text { apreciado }\end{array}$ & Bajo & Medio & Alto & Extremo & Activos & INACTIVOS & TOTAL & VICTIMAS & $\begin{array}{c}\text { PSP } \\
\text { desde } \\
17 / 08 / 2016\end{array}$ & $\begin{array}{c}\text { Gestión } 3 A \\
\text { desde } \\
15 / 02 / 2017\end{array}$ \\
\hline Cuerpo Nacional de Policía & 11.994 & 11.165 & 2.168 & 82 & 9 & 25.418 & 211.734 & 237.152 & 219.835 & 34.913 & 31.278 \\
\hline Policía Foral Navarra & 182 & 141 & 25 & & & 348 & 1.032 & 1.380 & 1.279 & 237 & 169 \\
\hline U.A. de Galicia & 72 & 135 & 21 & & & 228 & 240 & 468 & 404 & 224 & 60 \\
\hline PL Andalucía & 936 & 777 & 159 & 4 & & 1.876 & 3.836 & 5.712 & 5.113 & 1.680 & 1.083 \\
\hline \multicolumn{12}{|l|}{ PL Aragón } \\
\hline PL Asturias & 156 & 162 & 38 & 3 & & 359 & 1.378 & 1.737 & 1.534 & 410 & 462 \\
\hline PL Baleares & 102 & 90 & 18 & & & 210 & 1.007 & 1.217 & 1.071 & 123 & \\
\hline \multicolumn{12}{|l|}{ PL Cataluña } \\
\hline PL Comunidad Valenciana & 747 & 711 & 116 & 4 & & 1.578 & 3.998 & 5.576 & 4.859 & 1.702 & 860 \\
\hline \multicolumn{12}{|l|}{ PL Extremadura } \\
\hline PL Galicia & 117 & 69 & 13 & 1 & & 200 & 780 & 980 & 860 & 128 & 306 \\
\hline PL Madrid & 1.540 & 763 & 141 & 9 & & 2.453 & 7.208 & 9.661 & 8.791 & 2.163 & 1.293 \\
\hline \multicolumn{12}{|l|}{ PL Murcia } \\
\hline PL Navarra & 123 & 54 & 6 & & & 183 & 505 & 688 & 623 & 71 & 108 \\
\hline \multicolumn{12}{|l|}{ PL País Vasco } \\
\hline PL La Rioja & 15 & 20 & 51 & 6 & & 92 & 316 & 408 & 371 & 113 & 87 \\
\hline PL Ceuta & 78 & 67 & & & & 145 & 58 & 203 & 185 & 27 & 32 \\
\hline PL Melilla & 14 & 15 & 2 & & & 31 & 61 & 92 & 89 & 29 & 30 \\
\hline TOTAL & 27.869 & 22.699 & 4.566 & 175 & 10 & 55.319 & 365.922 & 421.241 & 391.856 & 68.287 & 57.028 \\
\hline \multicolumn{4}{|c|}{ Casos Mossos d'Esquadra enviados (vía servicios web) desde Cataluña } & & & \multicolumn{2}{|c|}{ CASOS BAJA } & 52.593 & & & \\
\hline
\end{tabular}

(1) Los casos inactivos de Cataluña y el País Vasco reflejan el número de víctimas que, estando recogidas en el Sistema VioGén (en otra parte del territorio nacional), en la actualidad residen en alguna de estas dos Comunidades Autónomas.

¿Cómo es posible que nos "ampare" un sistema que nos cataloga y designa en términos de "riesgo bajo" "riego medio" - como los estaba el caso de Andrea- o "no apreciado"? ¿"casos inactivos"? ¿"víctimas"?

Como señalan Sonia Núñez Puente et al. en su artículo 'Online feminist practice, participatory activism and public policies against gender-based violence in Spain' (2017), la significación por el discurso legal de las mujeres como víctimas es ineludible como parte del proceso para acceder a protección y asistencia institucional.

This situation also shows how women who experience partner-based violence in Spain are categorised as victims by legal discourse and, as shown above, it is only through assuming this label that they can access the assistance and protective mechanisms put into place by public policies, foreclosing possibilities for political resistance (Núñez Puente, 2017, p. 18). 
Muy lejos de lo que podrían ser para las mujeres instrumentos políticos de resistencia, espacios de participación o modos de recuperar su agencia y autonomía. Aquí se nos hace muy pertinente la plataforma Andrea.

Y es que en la búsqueda de espacios en Internet de apoyo a mujeres amenazadas por la violencia machista, nos encontramos con que las páginas web toman a su vez el discurso público y los debates gubernamentales, sin proponer apenas participación o interacción alguna por parte de las mujeres. Recalca Núñez Puente que tampoco el ciberactivismo feminista en España ha producido aún un discurso alternativo para la violencia de género frente al que se observa en las páginas webs institucionales:

Thus, we shall see how the webpages we analyze have an eminently static character and do not promote interaction or dialogue as a form of agency for activists and those who experience gender-based violence. This, as the results of our survey will show, is exactly what the surveyed women demand (Núñez Puente, 2017, p. 5).

Esto es lo que Andrea propone: “(...) ensuring that they are more effective and affective" (Núñez Puente, 2017, p. 19).

En todo caso es preciso matizar que la red Andrea no trata de suplantar la labor del Estado, la policía o los jueces, sino de hacer lo que ellos no pueden o ni siquiera conciben: generar tejido social y auto-organización.

\section{Arquitectura de la red y tareas en marcha}

Actualmente estamos trabajando en dar forma y contenido a la plataforma Andrea, sus diferentes bloques, posibilidades y recorridos dentro de la red. A continuación sintetizamos de manera más esquemática los distintos apartados de la red que, en parte, hemos ido explicando.

\subsection{Para todos/as los/as que queremos "hacer algo"}

Qué podemos hacer y qué nos ofrece la red.

- INSCRIBIRSE. La posibilidad de inscribirse en la red aportando un teléfono de contacto y un código postal para saber por dónde andamos cada cual. Darte de alta supone que te avisaremos cuando haya un caso de violencia machista cerca de tu casa y que pasarás a ser parte del grupo de apoyo de esa persona. También puedes inscribirte si eres una mujer que temes estar entrando en una situación de riesgo y quieres poder aprender más sobre tu propia circunstancia y la de otras mujeres.

- FORMARSE. La red organizará talleres de formación presenciales, así como cursos MOOC (gratuitos y virtuales) para que puedas dar una asistencia más cualificada en términos legales, psicológicos o de servicios y recursos sociales.

- ACTUAR. Los grupos de apoyo se activarán automáticamente cuando cualquier mujer así lo solicite seleccionando a las personas más próximas al domicilio de la mujer amenazada, que deberán confirmar su disponibilidad hasta lograr estabilizar una red de entre 4 y 12 personas. 
- IMPLICARSE MÁS. Las personas que así lo deseen podrán implicarse más trabajando para ampliar la red, los recursos formativos y sobre todo ejercer como supervisoras de grupos, constatando el buen funcionamiento de los grupos que le correspondan y mejorando su composición cuando así sea preciso.

\subsection{Cuando hay una mujer amenazada}

Cualquier mujer amenazada puede inscribirse como participante en redes de apoyo ya activas o puede, obviamente, solicitar la formación de un grupo de apoyo específico para ella, compuesto por gente cercana y que tenga el perfil y la formación más acorde con la situación en que ésta se encuentre. Por supuesto, esto no es excluyente de seguir participando en la red respaldando a otras mujeres, formándose, etc. Además, le ofrece un protocolo de privacidad para casos que así lo requieran (seguir viviendo con la pareja, etc.).

\subsection{Para la mujer amenazada}

- CONFIANZA. La posibilidad de encontrar apoyo inmediato, personalizado y próximo en un plano psicológico, legal o de recursos sociales. La mujer amenazada no estará aislada. Una vez se genera el "grupo de proximidad" de esta mujer, es ella la que lo administra y decide.

- ACOMPAÑAMIENTO. Si la mujer amenazada lo necesita y quiere, las personas del grupo pueden estar presentes para acompañarla. Acompañarla para ir al trabajo, para gestionar asuntos legales, para recoger a los niños, etc. También la posibilidad de alojamiento nocturno, o ayudarla a encontrar y afianzar otro entorno.

- ACCIÓN. Acciones directas siempre dirigidas por la mujer amenazada. Denuncia, presión a jueces, difusión vecinal...

\section{Infraestructura de la red}

Servidor donde se alojará una plataforma online (wordpress) y un BOT, es decir un servicio automatizado que ejecute operaciones como la asignación de personas a la red personal de cada mujer amenazada.

Esta plataforma contendrá los siguientes servicios o funcionalidades:

- Red social (ya instalada).

- Información general.

- Vídeos explicativos.

- Descarga de programas para la gestión de grupos a través del teléfono. Recurriremos a la aplicación Telegram, por tratarse de un software en abierto que nos permite un completo control de los desarrollos necesarios.

- Registro de usuarios: El registro se realizará mediante número de teléfono, calle y número y código postal, para posteriormente generar grupos automáticos dependiendo de la cercanía entre los usuarios. 
Desarrollos a realizar Wordpress:

- Maquetación de plataforma con el estilo y contenidos adecuados.

- Foros de discusión integrados con el resto de la plataforma.

- Botón que al pulsarlo elimine todo el historial, cookies, etc. del navegador del usuario/a.

- Foros de discusión.

- Valoraciones de los usuarios: si un usuario tiene $\mathrm{x}$ valoraciones negativas se ejecuta un BOT -un agente automatizado-que lo elimina de la plataforma e impedirá que pueda volver a registrarse.

Solicitud de ayuda:

- Los grupos se crearán una vez que uno de los usuarios genere una alarma: Mediante la api de Telegram se han de generar grupos automáticos y, dependiendo del tipo de alarma, el sistema BOT crea en Telegram un tipo de grupo.

- También la persona que genera la alarma elige el tipo de grupo que desea (femenino, mixto).

- La persona que genera la alarma pasa a ser la administradora del grupo, por lo que podrá eliminar miembros.

Desarrollos a realizar en Telegram:

- Generación de grupos en Telegram para agilizar la comunicación en casos necesarios.

- Instalación del BOT de asignación de personas a cada una de las redes de confianza.

- Interacción entre la plataforma (wordpress) y servicio BOT, mediante JSON

- Seguridad en Telegram: En el Telegram de la administradora, que será siempre la mujer maltratada o la persona designada por ella, se deberá contar no sólo con la posibilidad de administrar los miembros de la red, sino que dotará también de la capacidad de eliminar la conversación sin que quede rastro de la misma en ningún historial.

\section{Algunas consideraciones adicionales}

Como consecuencia de la discusión del proyecto en muy diferentes entornos e incluso tras el proceso de evaluación que ha sufrido este texto nos vemos en la tesitura de añadir algunas observaciones de capital importancia que quizás no hayan quedado suficientemente destacadas más arriba:

1. El proyecto no se dirige a mujeres amenazadas "por un lado" y a personas que puedan ayudarlas "de otro lado": uno de nuestros objetivos fundamentales es contribuir a la inteligencia de que las dinámicas de fragmentación, soledad e impotencia a las que aboca la violencia machista no afectan sólo a las mujeres amenazadas, sino que nos degradan y nos afectan a todos/as. 
Por ello, Andrea se presenta como una red de personas que se plantan ante esta situación y deciden organizarse para hacerle frente con independencia, en principio, de cual sea su situación particular.

2. Dicho esto el proyecto tiene muy en cuenta que cualquiera de las mujeres que forman parte de la red puede encontrarse ella misma en una situación de riesgo y requerir por tanto que se organice una red específica de confianza, acompañamiento y acción con la que sepa que puede contar. Toda vez que esa situación de riesgo haya sido atenuada o desactivada por completo, la mujer amenazada podrá seguir siendo parte de las redes de otras mujeres aportando su irreemplazable sensibilidad y experiencia.

3. La clave del proyecto, en consecuencia, es la auto-organización. Este será un aspecto crucial en la campaña de medios de comunicación y redes sociales que acompañará al lanzamiento de la iniciativa. Andrea no es más que una herramienta más dirigida a suscitar y sostener procesos de auto-organización.

4. Somos muy conscientes de que la cuestión de la seguridad es una de las transversalidades del proyecto a las que mayor atención habrá que dedicar. La elección misma de la aplicación Telegram se debe en gran medida a las posibilidades que tiene en ese sentido, permitiendo opciones como el borrado automático de conversaciones o el establecimiento de diferentes niveles de privacidad. Como es forzoso en un proyecto de estas características, la cuestión de la seguridad será un objeto permanente de investigación y mejora. En el momento de redacción de este texto nos encontramos apenas en los estadios iniciales del trabajo de programación y organización.

\section{Coda}

En el nombre de Andrea, tan irónica como pertinentemente parece resonar el antiguo concepto socrático de la "andreia". La "andreia" era para los antiguos el valor que hace a alguien digno de ser un hombre. Lo interesante es que en Sócrates la "andreia" se desvincula programáticamente de la fuerza bruta, así como de cualquier orden de violencia o sumisión que no sea la de las propias pasiones y temores. 
Figura 7. Andrea. Fuente: elaboración propia

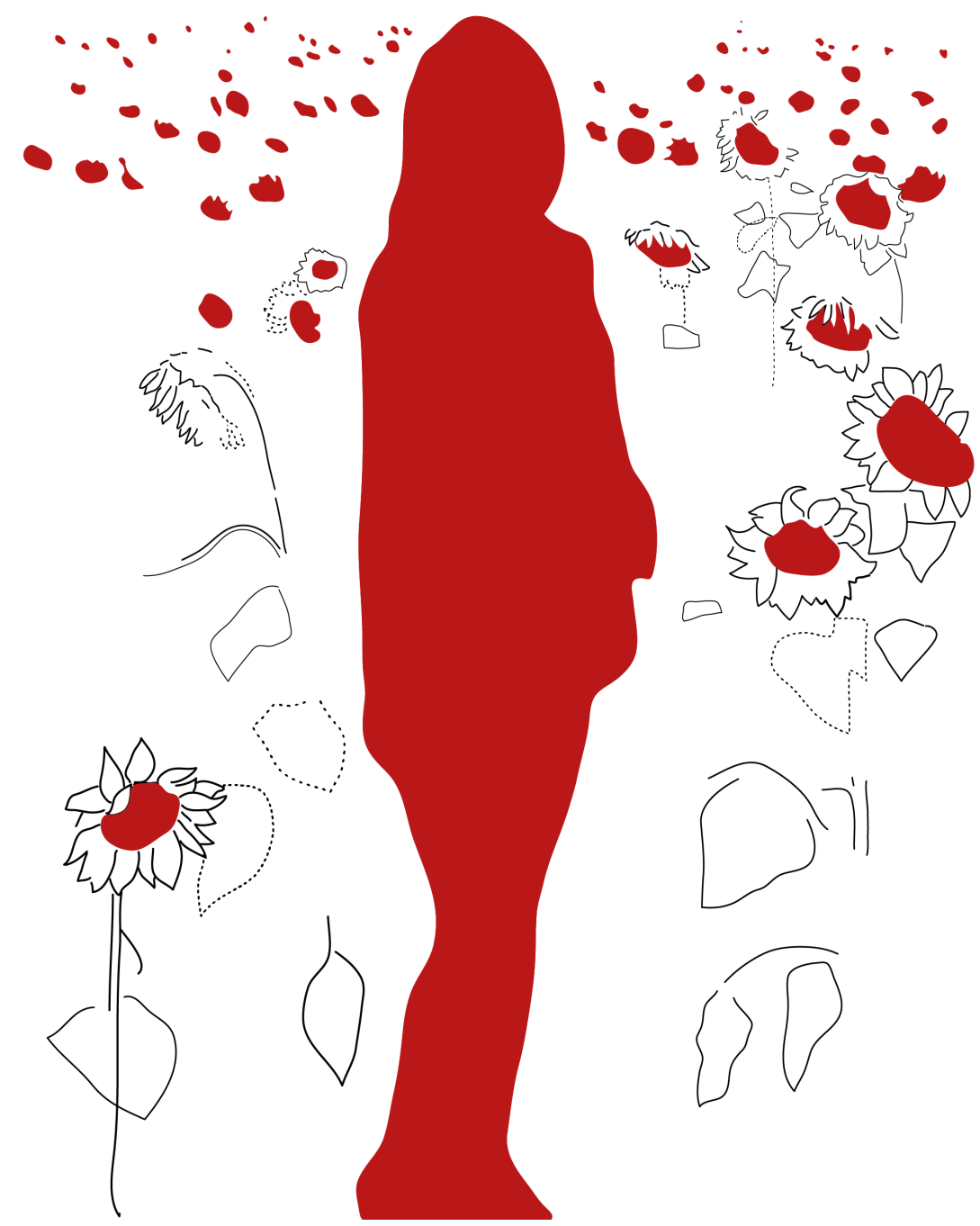

\section{Agradecimientos}

Andrea es un proyecto colectivo que sólo en colectivo ha podido ser pensado y empezar a desarrollarse. En este sentido es fundamental destacar que las personas que firmamos este articulo somos sólo responsables de la "redacción" del mismo, siendo así que todas y cada una de las ideas son el producto de meses de trabajo en los que personas como Elena Lenguas, Sergio Martín, Sonia Núñez Puente, Lidia Fernández y Alba Pérez han sido imprescindibles. Para ellas nuestro reconocimiento. 


\section{Referencias}

De Miguel Álvarez, A. (2005). La construcción de un marco feminista de interpretación: La violencia de género. Cuadernos de Trabajo Social, 18, 231-248.

Fernández Romero, D. (2012). Destrucción y Reconstrucción de la Identidad de la mujeres maltratadas: Análisis de discursos autobiográficos y de publicidad institucional. Memoria para optar al grado de doctor. Universidad Complutense de Madrid.

Ministerio de la Presidencia (s/f). Web informativa sobre violencia de género. Recuperado de: http://www.violenciagenero.igualdad.mpr.gob.es

Núñez Puente, S., Fernández Romero, D. y Vázquez Cupeiro, S. (2017). Cyberfeminism, Participatory Activism, and Public Policies against Gender-Based Violence in Spain, Feminist Theory, 18(3), 299-321. DOI: 10.1177/1464700117721881 